\title{
Towards a solution concept for network formation games
}

\author{
Andrea Gallo ${ }^{\mathrm{a}}$, Claudia Meroni ${ }^{\mathrm{b}, *}$ \\ ${ }^{a}$ School of Economics and Finance, Queen Mary University of London, London, United Kingdom. \\ ${ }^{b}$ Department of Economics, Management and Quantitative Methods, University of Milan, Milan, Italy.
}

\begin{abstract}
Network formation games (Myerson, 1991) typically present a multiplicity of Nash equilibria. Some of them are such that mutually beneficial links are not formed, thus inducing networks that are not pairwise stable. We offer an equilibrium refinement for this class of games which naturally involves pairwise stability while guaranteeing admissibility.
\end{abstract}

Keywords: Network formation games, equilibrium refinement, pairwise stability.

JEL Classification: C62, C72, D85.

\section{Introduction}

Network formation games are designed to understand ${ }^{35}$ how individuals' strategic incentives influence the structure of the network connecting them. In the simultane5 ous link-announcement game by Myerson (1991), players choose the set of links they wish to form and a link between two players is formed only if announced by both of them. This double-consent requirement generates a multiplicity of Nash equilibria. In some of them, couples of players do 10 not announce a link even if it would make them better off since each player is indifferent to the own unilateral deviation. The concept of pairwise stability introduced by Jackson and Wolinsky (1996) captures such a coordination aspect by requiring that every mutually beneficial link be

15 formed. Pairwise stable networks are not necessarily equilibrium outcomes of network formation games and, when they are, they may be induced by unplausible Nash equilibria. We offer an equilibrium refinement for this class of games that satisfies a desirable condition for strategic 20 stability and easily involves the coordination requirement of pairwise stability.

Some studies have been developed in order to pro- $^{55}$ vide a non-cooperative foundation of pairwise stability via solution concepts of network formation games. Calvó-

25 Armengol and İlkılıç (2009) consider the concept of proper equilibrium (Myerson, 1978), and furnish sufficient conditions on players' payoff functions for a proper equilibrium to imply pairwise stability and for a pairwise stable network to be induced by a proper equilibrium. [Ilkılıç and ${ }^{60}$ 30 İizler (2019) introduce the concept of trial perfect equilibrium, which requires less stringent conditions in the second direction. But this comes at the expense of admissibility, that is, the principle prescribing players not to play dominated strategies (Luce and Raiffa, 1957). Considering such a principle a basic desideratum for solution concepts of non-cooperative games, we propose a refinement which guarantees it like properness, and involves less-demanding requirements to capture pairwise stability than both the previous concepts.

We consider a refinement of Selten (1975)'s tremblinghand perfection that puts further restrictions on the converging sequence of strategies, requiring strategy perturbations to be independent across link announcements. This seems reasonable since we focus on pure strategy equilibria and interpret perturbations as mistakes that players do when announcing the links they wish to form. Roughly speaking, we limit the probability of making mistakes on a set of announcements to be of smaller order than the probability of making mistakes on any of its subsets ${ }^{1}$ We show that such a refinement, that we call b-perfect equilibrium, induces a pairwise stable network whenever players are not indifferent to the addition of a direct link. As we will see throughout the discussion, the intuitiveness of $b$-perfection translates also in a computational advantage relative to other concepts.

Section 2 presents the model, Section 3 discusses pairwise stability, Section 4 examines $b$-perfection, Section 5 concludes.

\section{The model}

Let $N=(1, \ldots, n)$ be the set of players. A network $g$ is a collection of links connecting them. We let $i j$ denote the link between player $i$ and player $j$ and we consider undirected networks, that is, for every link $i j$ we have $j i=$ $i j$. We let $g^{N}$ be the complete network in which each player ${ }_{65}$ is linked with every other player. We call the network

\footnotetext{
${ }^{1}$ For an application of an analogous solution concept to a different class of games, see De Sinopoli and Meroni (2018).
} 
where no link is formed the empty network. The power set ${ }_{115}$ of $g^{N}, \mathscr{G}=\left\{g \subseteq g^{N}\right\}$, is the set of all possible networks. The network payoff function $v: \mathscr{G} \rightarrow \mathbb{R}^{n}$ specifies for every network the payoff each player obtains.

70 A network formation game (Myerson, 1991) is a normal form game $G=(N, S, u)$. Each player $i \in N$ chooses 120 the links she wants to form. A pure strategy of player $i$ can be described as an $n$-1-dimensional vector $s_{i}=$ $\left(s_{i}^{1}, \ldots, s_{i}^{i-1}, s_{i}^{i+1}, \ldots, s_{i}^{n}\right)$ with entries $s_{i}^{j} \in\{0,1\}$ for ev75 ery $j \in N \backslash\{i\}$, where $s_{i}^{j}=1$ if player $i$ announces the link with player $j$ while $s_{i}^{j}=0$ if she does not. The set of ${ }^{125}$ player $i$ 's pure strategies is $S_{i}$, and the set of pure strategy profiles is $S=\prod_{i \in N} S_{i}$. The profile $s \in S$ induces the network $g(s)$ where a link between two players exists whenever

80 both of them announce it. The payoff function $u: S \rightarrow \mathbb{R}^{n}$ maps each strategy profile to the payoff that each player gets given the induced network, i.e. $u(s)=v \circ g(s)$.

Throughout the paper we will focus on Nash equilibria130 in pure strategies. A network $g$ is a Nash equilibrium net${ }_{85}$ work if there exists a Nash equilibrium $s$ of the network formation game that induces it, i.e. $g=g(s)$.

\section{Pairwise stability}

We begin by recalling the concept of pairwise stability and then discuss the drawbacks of pairwise stable networks

90 in terms of strategic stability of the strategy profiles they are supported by.

Definition 1. A network $g$ is pairwise stable with respect to the network payoff function $v$ if, for every $i, j \in N$,

(i) if $i j \in g$ then $v_{k}(g) \geq v_{k}(g \backslash\{i j\})$ for $k=i, j$;

(ii) if ij $\notin g$ then $v_{i}(g \cup\{i j\})>v_{i}(g)$ implies $v_{j}\left(g \cup_{145}\right.$ $\{i j\})<v_{j}(g)$.

Clearly, a Nash equilibrium network satisfies condition (i) in the above definition but does not necessarily satisfy condition (ii). On the other hand, a pairwise stable net100 work is not necessarily supported by a Nash equilibrium, ${ }_{150}$ as a player could be better off by cutting several of her links. It is standard to require that the strategy profile inducing a pairwise stable network be a Nash equilibrium, and if such a requirement is satisfied the network is called a pairwise-Nash equilibrium network.

From a strategic stability viewpoint, the Nash equilib-155 rium concept is unsatisfactory as it does not guarantee admissibility. Likewise, pairwise-Nash equilibrium networks may be induced by dominated Nash equilibria. Consider for instance three players, each of whom is indifferent between having no link or having a link with one of the others, but strictly prefers being linked with both of them. Announcing both links is a weakly dominant strategy for everyone $2^{2}$ However, the empty network is pairwise stable

\footnotetext{
2 Strictly dominated strategies do not appear in network formation games in which link formation requires mutual consent, and henceforth we will refer to weak dominance simply as dominance.
}

and is induced by multiple Nash equilibria, which are all dominated since at least two players are choosing a dominated strategy ${ }^{3}$

It is well-known that admissibility is captured by the concept of trembling-hand perfection and, obviously, is assured by every of its refinements. Calvó-Armengol and Ilkılıç (2009) apply the concept of proper equilibrium to network formation games and provide a sufficient condition for it to induce pairwise stable networks. In the next section we present a different refinement of perfect equilibrium, which involves pairwise stability under a milder condition.

\section{B-perfection}

First, we introduce some additional definitions. Let a $b$-strategy $b_{i}$ of player $i$ be an $n$-1-dimensional vector with entries $b_{i}^{j} \in[0,1]$ for every $j \in N \backslash\{i\}$, where $b_{i}^{j}$ represents the probability that player $i$ announces the link with player $j$. The set of $b$-strategies of player $i$ is $B_{i}$. A $b$-strategy profile $b$ is an element of $B=\prod_{i \in N} B_{i}$. Let $b(s)$ be the $b$ strategy profile corresponding to the pure strategy profile 35

A perfect equilibrium is the limit point of a sequence of completely-mixed strategy profiles that is best reply to every element of the sequence. We now define a refinement of perfect equilibrium, which requires the profiles in the converging sequence to be completely-mixed $b$-strategy profiles (i.e., such that $b_{i}^{j}>0$ for every $i$ and $j$ ).

Definition 2. The pure strategy profile $s$ is a b-perfect equilibrium of the network formation game if there exists a sequence of completely-mixed b-strategy profiles converging to $b(s)$ such that $s$ is best reply to every element of the sequence.

A network $g$ is a b-perfect equilibrium network if there exists a $b$-perfect equilibrium of the network formation game that induces it. The following example shows that not every pairwise-Nash equilibrium network is $b$-perfect, and viceversa.

Example 1. Consider a three-player network formation game, where payoffs of player 1 (at the top), player 2 (at the lower left), and player 3 (at the lower right) are as follows.

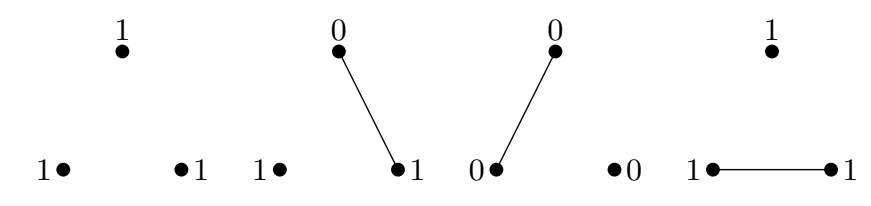

\footnotetext{
3 See also Example 1

4 The term b-strategy reminisces behavioral strategy. Note that the set of mixed strategies of player $i$ is $\Sigma_{i}=\Delta\left(S_{i}\right)$ and the set of mixed strategy profiles is $\Sigma=\prod_{i \in N} \Sigma_{i}$. Both mixed and $b$-strategy profiles induce probability distributions over $\mathscr{G}$.
} 

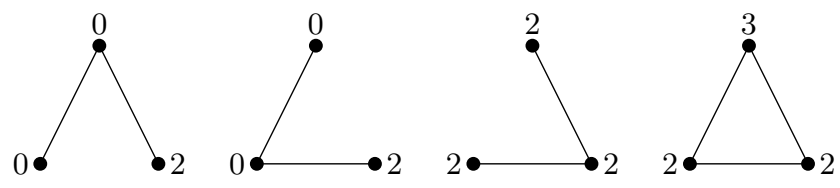

160

t is easy to see that the empty network is pairwise stable and is induced by several Nash equilibria (e.g., the profile in which no player announces any link). But none of them is b-perfect, since player 2 and player 3 have dominant 210 strategies, $s_{2}=(0,1)$ and $s_{3}=(1,1)$.

On the other hand, the network in which only link 12 is missing is b-perfect, being induced by the b-perfect equilibrium $s=((1,1),(0,1),(1,1)){ }^{5}$ However, it is not pairwise stable because the addition of link 12 makes player $1^{215}$ strictly better off but leaves player 2 indifferent, violating condition (ii) in Definition 1 .

In this example, the reason why the $b$-perfect equilibrium network is not pairwise stable is that player 2 is indifferent between having the link with player 1 or not, given the other links in the network. We define a condition on players' payoff functions, that we call local link responsiveness, which precisely requires that players be not indifferent if a single link of theirs is added to the network.

Definition 3. The network payoff function $v$ is locally link responsive on $g$ if $v_{i}(g \cup\{i j\}) \neq v_{i}(g)$ for all $i, j \in N$ and ij $\notin g$.

We can show that, under local link responsiveness, $b-230$ perfection readily implies pairwise stability.

Proposition 1. Let $g$ be a b-perfect equilibrium network. If $v$ is locally link responsive on $g$, then $g$ is pairwise stable.

Proof. Let $s$ be a $b$-perfect equilibrium and $g=g(s)$. Since every $b$-perfect equilibrium is a Nash equilibrium, we need to show that $g$ satisfies condition (ii) in Definition 1 . Con- ${ }^{235}$ sider a link $i j \notin g$ such that $v_{i}(g \cup\{i j\})>v_{i}(g)$. For the link not to be formed in equilibrium, it must be $s_{j}^{i}=0$.

Since $s$ is a $b$-perfect equilibrium, we have $s_{i}^{j}=1$. In fact, in any completely-mixed $b$-strategy profile close to $b(s)$, the probability that $s_{j}^{i}=1$ and all the other $\operatorname{link}^{24}$ announcements are as prescribed by $s$ is of higher order than the probability that $s_{j}^{i}=1$ and also other announcements change. By local link responsiveness, we have $v_{j}(g \cup\{i j\}) \neq v_{j}(g)$. If $v_{j}(g \cup\{i j\})>v_{j}(g)$ then player $j$ 's best reply against $s$ would prescribe $s_{j}^{i}=1$, so it must be $v_{j}(g \cup\{i j\})<v_{j}(g)$, as required by pairwise stability.

5 Note that in any completely-mixed $b$-strategy profile close to $b(s)$, the probability that $s_{2}^{1}=1$ and all the other announcements are as prescribed by $s$ is infinitely larger than the probability that $s_{2}^{1}=1$ and also other announcements change, so player 1 's unique best reply is $s_{1}=(1,1)$.
Calvó-Armengol and İlkılıç (2009) show that proper equilibria induce pairwise stable networks under strong link responsiveness, a condition requiring players not to be indifferent to any change in their set of direct links. They offer an example where that condition is not satisfied and a proper equilibrium network fails to be pairwise stable. We can consider that same example to see how $b$-perfection outperforms properness in capturing pairwise stability (we refer to the example "Violation of strong link responsiveness" in Calvó-Armengol and Ilkılıç, 2009. p.74) ${ }^{6}$ In fact, $b$-perfection rules out the unfortunate network $g_{0}$ that is induced by the proper equilibrium $s^{*}$. In any completely-mixed $b$-strategy profile close to $b\left(s^{*}\right)$, indeed, the probability that player 4 announces the link with player 3 and the other announcements are as prescribed by $s^{*}$ is of higher order than the probability that $s_{4}^{3}=1$ and also other announcements change. Thus, it is a best reply for player 3 to announce also the link with player 4 . Since an analogous and symmetric reasoning holds for player 4, $g_{0}$ is not induced by any $b$-perfect equilibrium.

We note that also the concept of trial perfect equilibrium of İkılıç and İkizler (2019) supports the bad network $g_{0}$, as any proper equilibrium is trial perfect.7 Moreover, its definition allows the strategy profiles in the converging sequence to not be completely-mixed, thus not guaranteeing undominance 8

The computational advantage of $b$-perfection emerges also when analyzing whether a pairwise-Nash equilibrium network satisfies it. In particular, given such a network and under local link responsiveness, it is enough to consider the strategy profile in which every player announces her existing links and those whose addition makes her better off. If such a profile is a Nash equilibrium supporting the network under consideration, then this is $b$-perfect.

\section{Conclusions}

We have introduced an equilibrium refinement for network formation games which is motivated by the actual procedure of announcing links. It outperforms other refinements proposed by the literature in inducing pairwise stability, while satisfying the basic requirement of admissibility. The question naturally arises as to how such a

${ }^{6}$ We make explicit that when players 3 and 4 have no direct links their payoffs must be lower than or equal to zero for $g_{0}$ (the network in which only links 13 and 24 are formed) to be a Nash equilibrium network. We assume that in that case they get -1 , which ensures that $g_{0}$ is a proper equilibrium network, being induced by the same proper equilibrium $s^{*}=((0,1,1),(0,1,1),(1,0,0),(0,1,0))$.

7 That concept requires the converging sequence of strategy profiles to assign strictly positive probability to all best replies, and orders perturbations so that more costly mistakes are made with lower order probability.

8 Consider, for instance, the example "Violation of $\beta$-strong supermodularity" in Calvó-Armengol and Illkılıç (2009 p.77). The pairwise-Nash equilibrium network $g^{\prime \prime}$, which is induced only by dominated equilibria, is trial perfect, as the condition of strong link responsiveness required by İlkılıç and İkizler (2019) is satisfied. 
solution concept relates to a context-independent theory of strategic stability in network formation games.

\section{Declaration of competing interest}

The authors declare that they have no known competing financial interests or personal relationships that could have appeared to influence the work reported in this paper.

\section{Acknowledgements}

We thank the editor and an anonymous referee for useful comments. We are grateful to Francesco De Sinopoli for helpful discussions. Financial support from the Italian Ministry of Education, PRIN 2017, grant number 2017K8ANN4 is acknowledged. The usual disclaimer applies.

\section{References}

Calvó-Armengol, A., İlkılıç, R. Pairwise-stability and Nash equilibria in network formation. Internat. J.

n Game Theory. 38:51-79, 2009. https://doi.org/10.1007/ s00182-008-0140-7

De Sinopoli, F., Meroni, C. A concept of sincerity for com260 binatorial voting. Soc. Choice and Welf. 51:493-512, 2018. https://doi.org/10.1007/s00355-018-1125-5

Ilkılıç, R., Ikizler, H. Equilibrium refinements for the network

11 formation game. Rev. Econ. Des. 23:13-25, 2019. https: //doi.org/10.1007/s10058-019-00218-y

265 Jackson, M.O., Wolinsky, A. A strategic model of social and 11 economic networks. J. Econ. Theory. 71:44-74, 1996. https: //doi.org/10.1006/jeth.1996.0108

Luce, R.D., Raiffa, H. Games and decisions: Introduction and critical survey. Courier Dover Publications, 1957. https: $270 \quad / /$ doi.org/10.2307/2342906

Myerson, R.B. Game Theory: Analysis of Conflict. Har-

n vard University Press, 1991. https://doi.org/10.1002/ mde.4090130412

Myerson, R.B. Refinements of the Nash equilibrium concept. 275 Internat. J. Game Theory. 7:73-80, 1978. https://doi.org/ 10.1007/BF01753236

Selten, R. Re-examination of the perfectness concept for equilibrium points in extensive games. Internat. J. Game Theory. 4:24-55, 1975. https://doi.org/10.1007/BF01766400 